\title{
Psychological and health comorbidities before and after bariatric surgery: a longitudinal study
}

\author{
Comorbidades físicas e psicológicas antes e depois da cirurgia bariátrica: \\ um estudo longitudinal
}

\author{
Susana Sofia Pereira da Silva, ${ }^{1}$ Ângela da Costa Maia ${ }^{2}$
}

\begin{abstract}
Introduction: Morbid obesity has multiple implications for psychological and physical health. Bariatric surgery has been selected as the treatment of choice for this chronic disease, despite the controversial impact of the surgery on psychosocial health. The objective of this study was to describe candidates for bariatric surgery and analyze changes in weight, psychopathology, personality, and health problems and complaints at 6- and 12- month follow-up assessments.

Methods: Thirty obese patients ( 20 women and 10 men) with a mean age of $39.17 \pm 8.81$ years were evaluated in different dimensions before surgery and 6 and 12 months after the procedure.

Results: Six and 12 months after bariatric surgery, patients reported significant weight loss and a significant reduction in the number of health problems and complaints. The rates of self-reported psychopathology were low before surgery, and there were no statistically significant changes over time. The conscientiousness, extraversion, and agreeableness dimensions increased, but neuroticism and openness remained unchanged. All changes had a medium effect size.

Conclusions: Our results suggest that patients experience significant health improvements and some positive personality changes after bariatric surgery. Even though these findings underscore the role of bariatric surgery as a relevant treatment for morbid obesity, more in-depth longitudinal studies are needed to elucidate the evolution of patients after the procedure.
\end{abstract}

Keywords: Bariatric surgery, health problems, obesity, psychopathology.

\section{Resumo}

Introdução: $\mathrm{A}$ obesidade mórbida tem várias implicações para a saúde psicológica e física. A cirurgia bariátrica tem sido o tratamento de escolha para essa doença crônica, apesar da controvérsia sobre o impacto da cirurgia na saúde psicossocial. O objetivo deste estudo foi descrever candidatos a cirurgia bariátrica e analisar mudanças no peso, psicopatologia personalidade, problemas e queixas de saúde desses pacientes em avaliações realizadas 6 e 12 meses após a cirurgia.

Métodos: Trinta pacientes obesos (20 mulheres e 10 homens) com idade média de $39,17 \pm 8,81$ anos foram avaliados em diferentes dimensões antes da cirurgia e 6 e 12 meses após.

Resultados: Aos 6 e 12 meses após a cirurgia bariátrica, os pacientes relataram significativa perda de peso e significativa redução no número de problemas e queixas de saúde. As taxas de psicopatologia autorrelatada foram baixas antes da cirurgia e não sofreram mudanças significativas com o tempo. As dimensões conscienciosidade, extroversão e agradabilidade aumentaram, mas o neuroticismo e a abertura permaneceram inalteradas. Todas as mudanças apresentaram um tamanho de efeito médio. Conclusões: Os nossos resultados sugerem que os pacientes experimentam melhoras significativas em saúde e algumas mudanças positivas de personalidade após a cirurgia bariátrica. Embora esses achados reforcem o papel da cirurgia bariátrica como um tratamento relevante para a obesidade mórbida, mais estudos longitudinais e aprofundados são necessários para elucidar a evolução dos pacientes após a realização do procedimento. Descritores: Cirurgia bariátrica, problemas de saúde, obesidade, psicopatologia.

\footnotetext{
${ }^{1}$ School of Management and Industrial Studies, Portugal. ${ }^{2}$ Center for Research in Psychology, School of Psychology, University of Minho, Portugal. Financial support: Foundation for Science and Technology (protocol no. SFRH/BD/37069/2007).

Submitted Feb 28 2013, accepted for publication Jul 08 2013. No conflicts of interest declared concerning the publication of this article.

Suggested citation: da Silva SS, Maia AC. Psychological and health comorbidities before and after bariatric surgery: a longitudinal study. Trends Psychiatry Psychother. 2013;35(4):264-271. http://dx.doi.org/10.1590/2237-6089-2013-0007
} 


\section{Introduction}

Obesity is a chronic disease with increasing prevalence, and it has become a major public health problem in industrialized societies. ${ }^{1}$ In addition to directly affecting quality of life, obesity causes an increase in other health problems and creates several personal and interpersonal restrictions.

According to the World Health Organization ${ }^{1}$ and the International Obesity Task Force (Strategic Plan 2010), obesity has reached global epidemic proportions, with more than one billion adults classified as overweight and at least 300 million as obese. Half of the European population is reportedly overweight or obese. ${ }^{2}$ Obesity is primarily responsible for the increase of other chronic diseases and functional disabilities - which, together, have reduced life expectancy. The number of deaths attributable to obesity is a serious problem in Europe: $7.7 \%$ of all deaths are related to excess weight. ${ }^{3}$

The main obstacle for various proposed treatments is maintaining long-term weight loss. In this scenario, bariatric surgery has emerged as the most effective treatment, with the potential to avoid the limitations of other interventions. ${ }^{4}$

Obesity is associated with a decrease in quality of life and an increase in mortality rates. Obesity-related morbidity, in turn, is associated with an increasing probability of contracting several medical conditions. Epidemiologic studies ${ }^{5}$ have shown that obese individuals seek more health care services, have higher health care costs, and have more health problems than normal persons, especially cardiovascular diseases, type 2 diabetes, pulmonary problems, obstructive sleep apnea, cancer, and osteoarticular and skeletal problems.

Data from the Swedish Obese Study (SOS) ${ }^{6}$ showed that patients presenting comorbidities enjoyed several medical benefits after bariatric surgery. Two years after recovery, $73 \%$ of the surgically treated group no longer suffered from type 2 diabetes, compared to only $19 \%$ of the control group. Hypertension is another common problem among bariatric surgery candidates. Typically, hypertension is resolved or improved in $66 \%$ of all surgically treated patients. According to the SOS data, 2 years after surgery, hypertension was observed in $9.9 \%$ of the obese control group and in only $3.2 \%$ of the surgical group. However, after 8 years, these numbers rose to 25.8 and $26.4 \%$, respectively, suggesting that the advantages associated with surgery tend to decrease over time. Obstructive sleep apnea is another prevalent disease among these patients, associated with poor quality of life and reduced life expectancy. A 4-year follow-up study showed that the disease was significantly less frequent in the surgically treated group, while the situation remained unchanged in the control group. ${ }^{7,8}$

In addition to physical health characteristics and improvements after surgery, recent research has tried to understand the psychological profile of obese patients and the short- and long-term consequences of bariatric surgery in this field. For instance, Abilés et al. ${ }^{9}$ have recently concluded that obese patients (type III and type IV obesity) report a higher number of psychological and social problems, worse quality of life, and a greater tendency to eat in response to negative emotions and contextual cues.

With regard to the psychiatric impact of obesity, studies have provided conflicting results. On the one hand, several authors ${ }^{10,11}$ have reported that morbidly obese patients who seek bariatric surgery show more psychopathologies, including anxiety, mood disorders, and impulsive behaviors. On the other hand, some studies $^{12}$ have also suggested that the prevalence of psychopathologies in obese people (types III and IV) is not substantially different from that of the normal population. In fact, a European study involving 5,817 subjects found that obese persons reported less anxiety and depression than non-obese persons. ${ }^{9}$

No consensus exists in the literature regarding the impact of psychopathology on surgery success or on changes taking place after surgery. For some patients, psychopathology may increase the risk of suboptimal surgical outcomes, while for others it bears no relevance and no impact on surgical outcomes.

Mitchell \& Zwaan ${ }^{13}$ suggest that bariatric surgery has a positive short-term impact on psychological functioning that may dissipate over time. Also, Guisado et al. ${ }^{14}$ found that, 1 year after bariatric surgery, morbidly obese patients reported less distress and psychological discomfort and more self-control than they did prior to the surgery.

In addition to psychopathology, personality characteristics have also been given attention by researchers studying psychosocial issues related to bariatric surgery. Some decades ago, CastelnuovoTedesco ${ }^{15,16}$ suggested that morbidly obese people seeking treatment for obesity often demonstrate certain personality traits, such as passive dependency and passive aggressiveness; Bruch ${ }^{17}$ wrote that these patients may appear passive but express hostility in relationships. Moreover, according to other authors, ${ }^{10,18}$ obese adults suffer from immaturity, poor impulse control, insecurity, emotional instability, and eccentric cluster traits. In contrast, recent studies conducted in the United States ${ }^{19}$ and Europe ${ }^{5}$ have shown that the differences in personality between morbidly obese persons and the rest of the population are minimal. Similarly, in a previous study, we did not find any differences in personality traits when comparing morbidly obese and non-obese subjects. ${ }^{20}$ 
The heterogeneity of findings on personality traits ${ }^{10,21}$ among the morbidly obese and the lack of longitudinal studies assessing patients before and after bariatric surgery underscore the need for the development of studies to analyze these dimensions in greater detail. Specifically, the outcomes of bariatric surgery and the impact of this procedure on different personality dimensions are major topics of interest for professionals and investigators working in this field. Some studies have shown huge variability in the profile of bariatric surgery candidates and in surgery outcomes. However, aside from the variability associated with the procedure, very little is known about why some patients lose more weight than others or about the impact of surgery on quality of life and health.

Therefore, the objective of the present study was to characterize candidates for bariatric surgery and to analyze changes in weight, health problems and complaints, psychopathology, and personality, 6 and 12 months after the procedure, the hypothesis being that a decrease in weight, health problems and complaints would be found. For psychopathology, no hypothesis was made, based on both the scarcity of research findings and the lack of consensus in the few data available; for personality, our hypothesis was that there would be no changes, i.e., that these traits would remain stable.

\section{Methods}

The study included 30 (20 women and 10 men) patients seen at the Multidisciplinary Obesity Treatment Center in northern Portugal. All patients undergoing bariatric surgery between June 2008 and June 2009 were assessed. All participants were evaluated 1 month before surgery, and then at 6 and 12 months of follow-up. All evaluations were conducted after medical assessment. Table 1 shows the main sociodemographic characteristics of the sample.

Table 1 - Sociodemographic characteristics of the sample

\begin{tabular}{|c|c|c|c|c|}
\hline \multirow{2}{*}{ Variable } & \multicolumn{2}{|c|}{ Female } & \multicolumn{2}{|c|}{ Male } \\
\hline & Mean & SD & Mean & SD \\
\hline Age & 38.99 & 8.04 & 37.12 & 8.75 \\
\hline Weight at surgery $(\mathrm{kg})$ & 118.24 & 18.05 & 120.82 & 17.3 \\
\hline \multicolumn{5}{|l|}{ Weight at 6 months $(\mathrm{kg})$} \\
\hline Current weight & 123.43 & 13.9 & 124.19 & 14.2 \\
\hline Minimal weight & 84.7 & 13.22 & 87.23 & 14.21 \\
\hline Maximal weight & 89.99 & 14.03 & 90.0288 & 12.45 \\
\hline \multicolumn{5}{|l|}{ Weight at 12 months $(\mathrm{kg})$} \\
\hline Current weight & 85.36 & 15.23 & 88.17 & 12.34 \\
\hline Minimal weight & 81.27 & 12.43 & 85.45 & 12.45 \\
\hline \multirow[t]{2}{*}{ Maximal weight } & 122.43 & 9.5 & 115.556 & 12.05 \\
\hline & $\mathbf{n}$ & $\%$ & $\mathbf{n}$ & $\%$ \\
\hline Gender & 20 & 67 & 10 & 33 \\
\hline \multicolumn{5}{|l|}{ Marital status } \\
\hline Single & - & - & - & - \\
\hline Married & 18 & 60 & 10 & 33 \\
\hline Divorced & 2 & 7 & - & - \\
\hline \multicolumn{5}{|l|}{ Education } \\
\hline 4 years & 3 & 10 & 3 & 10 \\
\hline 9 years & 10 & 33 & 5 & 17 \\
\hline High school & 7 & 23 & 2 & 7 \\
\hline \multicolumn{5}{|l|}{ Professional status } \\
\hline Full time & 16 & 53 & 5 & 18 \\
\hline Part-time & 1 & 3 & 1 & 3 \\
\hline Unemployed & 2 & 7 & 3 & 10 \\
\hline Retired & 1 & 3 & 1 & 3 \\
\hline
\end{tabular}

$\mathrm{SD}=$ standard deviation . 
The study protocol was approved by the Ethics Committee of the hospital, and all participants signed an informed consent form before their inclusion in the study.

\section{Instruments}

Sociodemographic Questionnaire (Silva SS \& Maia AC, Sociodemographic Questionnaire, Portuguese edition, 2008): a self-report questionnaire collecting data on gender, age, education, weight, and marital and professional status.

Rotterdam Symptom Checklist (RSCL)22 (McIntyre T \& Gameiro J, Portuguese version of the Rotterdam Symptom Checklist - RSCL, 1997): a 30-item instrument that measures psychological and physical morbidity by analyzing the intensity of symptoms using a 4-point Likert scale. In this study, only the physical morbidity scale was used, and a new item (joint pain) was introduced given the specific characteristics of the obese population. This checklist has shown good psychometric properties (Cronbach's alpha $=0.91$ for the global scale, 0.86 for the physical morbidity scale, and 0.88 for the psychological morbidity scale, respectively)..$^{22}$ In this study, Cronbach's alpha for the physical morbidity scale was 0.85 .

Self-Report Diseases Checklist (Silva SS \& Maia AC, 2007): a list of 14 diseases, including hypertension, diabetes, and sleep apnea syndrome. This checklist yields a single index for all health problems reported by each individual.

Brief Symptom Inventory (BSI) ${ }^{23,24}$ : a 53-item self-report measure designed to evaluate general psychiatric morbidity. Subjects answer questions using a 5-point Likert scale ranging from 0 (never) to 4 (several times). The BSI yields a general symptom index. The Portuguese version has shown good psychometric qualities. ${ }^{23,24}$ Cronbach's alfa in this study was 0.94 .
NEO Five-Factor Inventory25: a 60-item self-report measure based on the Revised NEO Personality Inventory designed to evaluate personality traits using a five-factor model: extraversion, agreeableness, conscientiousness, neuroticism, and openness to experience. Subjects answer questions using a 5-point Likert scale ranging from 0 (strongly disagree) to 4 (totally agree). In this study, Cronbach's alpha was 0.87 for extraversion and conscientiousness, 0.88 for agreeableness and openness to experience, and 0.81 for neuroticism.

\section{Statistical analysis}

Data were analyzed using the Statistical Package for the Social Sciences (SPSS) version 18.0. Univariate and bivariate statistical analyses were performed. Prior to analysis, all continuous variables were assessed for normality using the Kolmogorov-Smirnov test, and normality criteria were not satisfied. The Friedman test was used to analyze changes in variables over time, and the Wilcoxon test was run to examine matched pairs for significant values. The least significant difference (LSD) test was used to control for type I errors. ${ }^{26}$ To compare our data on psychopathology with data from the study describing the Portuguese adaptation of the BSI, one-sample t tests were performed. Significance was set at $p<0.05$.

\section{Results}

\section{Weight}

Data in Table 2 show that mean weight before surgery was $122.43 \pm 14.11 \mathrm{~kg}$, compared to $89.69 \pm 13.88 \mathrm{~kg} 6$ months after surgery and $85.05 \pm 14.17 \mathrm{~kg}$ at 12 months. Significant differences were observed in the patients' median weight prior to surgery (median $=100.5$ ), at the

Table 2 - Descriptive statistic results for the dimensions assessed at the three evaluations and differences over time

\begin{tabular}{|c|c|c|c|c|c|c|c|}
\hline \multirow{2}{*}{ Variable } & \multicolumn{2}{|c|}{ Before surgery } & \multicolumn{2}{|c|}{ 6-month follow-up } & \multicolumn{2}{|c|}{ 12-month follow-up } & \multirow{2}{*}{ Friedman's $\chi^{2}$} \\
\hline & $\bar{\chi}$ & SD & $\bar{\chi}$ & SD & $\bar{\chi}$ & SD & \\
\hline Weight & 122.43 & 14.1 & 89.69 & 13.88 & 85.05 & 14.17 & $25.72 *$ \\
\hline Health complaints & 14.10 & 7.66 & 11.13 & 7.61 & 12.50 & 7.35 & $8.909^{+}$ \\
\hline Health problems & 4.2 & 1.18 & 3.1 & 1.4 & 2.9 & 1.4 & $9.254^{+}$ \\
\hline General psychopathology & 0.73 & 0.47 & 0.77 & 0.56 & 0.90 & 0.67 & 2.531 (ns) \\
\hline \multicolumn{8}{|l|}{ Personality } \\
\hline Neuroticism & 24.5 & 7.91 & 25.9 & 6.93 & 25.37 & 8.14 & 1.564 (ns) \\
\hline Openness & 22.47 & 4.32 & 22.16 & 4.24 & 23.1 & 4.93 & 0.442 (ns) \\
\hline Conscientiousness & 13.1 & 3.97 & 23.77 & 8.46 & 25.27 & 11.76 & $20.579 *$ \\
\hline Extraversion & 18.03 & 6.12 & 23.70 & 5.72 & 25.07 & 8.30 & $10.320^{+}$ \\
\hline Agreeableness & 15.77 & 4.36 & 20.67 & 6.54 & 22.63 & 8.64 & $13.604^{*}$ \\
\hline
\end{tabular}

ns $=$ non-significant; $\bar{\chi}=$ mean; SD = standard deviation.

${ }^{*} \mathrm{p}<0.001,{ }^{+} \mathrm{p}<0.01$. 
6-month follow-up evaluation (median $=88.3$ ), and at 12 months (median $=84.5)\left(\right.$ Friedman's $\chi^{2}[2, \mathrm{n}=30]=$ 25.717, $\mathrm{p}=0.000)$.

As shown in Table 3, following pairwise comparisons (Wilcoxon) and control for type I errors (LSD), median weight prior to surgery remained significantly greater than median weight at 6 months ( $p=0.000$, with an effect size of $r=0.498)$ and at 12 months ( $p=0.000$, $r=0.559)$. Notwithstanding, median weight at the 6-month follow-up evaluation did not differ from that at 12 months ( $p=0.174)$.

\section{Health problems and complaints}

The percentage of subjects reporting at least one problem before surgery was very high: $80 \%(n=24)$. The problems most frequently reported before surgery were hypertension ( $48 \%, \mathrm{n}=14)$, diabetes $(20 \%, \mathrm{n}=6)$, thyroid disease $(14 \%, n=4)$, sleep apnea $(12 \%, n=3)$, and gallstones $(12 \%, \mathrm{n}=3)$.

Six months after surgery, $60 \%(n=18)$ of the subjects reported at least one health problem. The problems most frequently reported then were hypertension ( $30 \%, n=9)$, diabetes $(10 \%, n=3)$, thyroid disease $(16 \%, n=4)$, sleep apnea $(8 \%, n=2)$, and gallstones $(16 \%, n=4)$.

Finally, at the 12-month follow-up evaluation, 50\% $(n=15)$ of the subjects reported the presence of at least one health problem, including hypertension $(20 \%, \mathrm{n}=6)$, gallstones $(12 \%, \mathrm{n}=3)$, thyroid disease $(16 \%, \mathrm{n}=3)$, diabetes $(8 \%, n=2)$, and sleep apnea $(4 \%, n=1)$.

The Friedman test indicated significant differences $\left(\chi^{2}\right.$ $[2, \mathrm{n}=30]=9.254, \mathrm{p}=0.01)$ in medians when comparing health problems prior to surgery (median $=3$ ), at 6 months ( median $=2$ ), and at 12 months (median $=1.5$ )

The analysis after the Friedman test showed that the median number of health problems was significantly higher prior to surgery than at the 6-month follow-up evaluation ( $p=0.01, r=0.331$ ) and at 12 months ( $p=0.014, r=0.319)$. However, the median number observed at 6 months did not differ significantly from the median observed at 12 months ( $p=0.336)$.

Mean number of health complaints (measured by the RSCL) at the time of surgery was $14.01 \pm 7.66$, vs. $13.11 \pm 7.61$ at 6 months and $12.5 \pm 7.35$ at 12 months.

There were differences in the median number of health complaints prior to surgery (median $=13$ ), at the 6-month follow-up evaluation (median $=9.5$ ), and at the 12-month follow-up test (median $=12$ ). The Friedman test resulted significant $\left(\chi^{2}[2, \mathrm{n}=30]=8.909, \mathrm{p}=\right.$ $0.01)$. Subsequent pairwise comparisons indicated that the median number of health complaints prior to surgery was significantly greater than 6 months after surgery ( $p$ $=0.01, r=0.312$ ). Conversely, the median number of complaints at 12 months did not significantly differ from the median obtained prior to surgery $(p=0.328)$ or at the 6 -month evaluation $(p=0.213)$.

\section{Psychopathology}

With regard to psychopathology, the mean scores obtained by our participants were very similar to those reported for the reference community sample in the Portuguese adaptation process. A one-sample $t$ test confirmed that the differences between our group results and reference data were not significant $(-1.221,-0.656$, and 0.462 , all non-significant). Conversely, the Friedman test (Table 2) showed that there were no significant changes in the patients' psychopathologies over time.

\section{Personality}

As shown in Table 2, there were no significant changes in the neuroticism and openness dimensions over time.

For the conscientiousness dimension, there were significant differences in median scores prior to surgery ( median $=13.5)$, at 6 months (median $=20.5$ ), and at 12 months $($ median $=26.5)\left(\chi^{2}[2, \mathrm{n}=30]=20.579, \mathrm{p}=\right.$ 0.000 ). The median conscientiousness score obtained prior to surgery was significantly lower than those obtained at the 6-month evaluation ( $p=0.000, r=0.477$ ) and at the

Table 3 - Descriptive statistic results (medians) for the dimensions assessed at the three evaluations and effect sizes over time

\begin{tabular}{lcccccc}
\hline Variable & $\begin{array}{c}\text { Before } \\
\text { surgery (t1) }\end{array}$ & $\begin{array}{c}\text { 6-month } \\
\text { follow-up (t2) }\end{array}$ & $\begin{array}{c}\text { 12-month } \\
\text { follow-up (t3) }\end{array}$ & \multicolumn{3}{c}{ Effect size } \\
\cline { 5 - 7 } Weight & 100.5 & 88.3 & 84.5 & $0.498^{*}$ & $0.175(\mathrm{~ns})$ & $0.559^{+}$ \\
Health complaints & 13 & 9.5 & 12 & $0.312^{+}$ & $0.160(\mathrm{~ns})$ & $0.126(\mathrm{~ns})$ \\
Health problems & 3 & 2 & 1.5 & $0.331^{+}$ & $0.124(\mathrm{~ns})$ & $0.319^{+}$ \\
$\begin{array}{l}\text { Personality } \\
\quad \text { Conscientiousness }\end{array}$ & 13.5 & 20.5 & & & & \\
\multicolumn{1}{l}{$\begin{array}{l}\text { Extraversion } \\
\text { Agreeableness }\end{array}$} & 18 & 23.7 & 26.5 & $0.477^{+}$ & $0.0169(\mathrm{~ns})$ & $0.450^{+}$ \\
\hline
\end{tabular}

ns $=$ non-significant.

$* \mathrm{p}<0.001,{ }^{+} \mathrm{p}<0.01$. 
12-month follow-up $(p=0.000, r=0.45)$. Notwithstanding, the median obtained at 6 months did not differ significantly from that of the 12-month evaluation ( $p=0.896$ ) (Table 3).

The Friedman test also revealed differences in the median scores obtained for the extraversion dimension $\left(\chi^{2}\right.$ $[2, \mathrm{n}=30]=10.32, \mathrm{p}=0.006)$. Using the same procedures described above, the median prior to surgery (median $=18$ ) was found to be significantly lower than that obtained at 6 months (median $=23.7, p=0.001, r=0.414$ ) and at 12 months (median $=25.1, p=0.004, r=0.376$ ). However, 6-month results did not differ significantly from 12-month data ( $p=0.822)$.

The same analysis also revealed significant differences in the median scores of agreeableness prior to surgery $($ median $=16)$, at 6 months (median $=19.5)$, and at 12 months $($ median $=20.5)\left(\chi^{2}[2, \mathrm{n}=30]=13.604\right.$, $\mathrm{p}=0.001)$. Pairwise comparisons and the LSD procedure showed that the median score obtained prior to surgery was significantly lower than the median obtained at 6 months ( $p=0.003, r=0.48)$ and at 12 months ( $p=$ $0.000, r=0.457)$. The median obtained at the 6 -month follow-up evaluation did not differ significantly from that of the 12-month follow-up ( $p=0.212)$.

\section{Discussion}

The results of our study suggest the existence of significant differences in physical and psychosocial characteristics before bariatric surgery vs. 6 and 12 months after the procedure. These differences include significant weight loss, a decrease in health problems and complaints, and an increase in the scores of some personality dimension, namely, conscientiousness, extraversion, and agreeableness.

We also found statistically significant differences in weight over time, with a medium effect size in the time period elapsed between the surgery and the 6-month follow-up evaluation ( $r=0.498)$, as well as a large effect size between the surgery and the 12-month follow-up visit $(r=0.559)$. Moreover, no significant differences were observed between the 6- and the 12-month follow-up evaluations. In accordance with the literature, 7,27 our results confirm that bariatric surgery is an effective way to lose excess weight, even though this loss tends to blur over time.

As stated in the literature, ${ }^{26}$ we also found a high prevalence of certain medical conditions often associated with obesity, as well as high morbidity and mortality related to this chronic disease. Nonetheless, patients experienced a moderate improvement of these medical conditions as early as 6 months after surgery. Thus, our results support the findings of the literature that associate bariatric surgery with the rapid improvement of medical comorbidities. . $^{6} 28-30$
Regarding psychopathologies, as already noted by some authors ${ }^{13}$ and denied by others, ${ }^{11,31}$ our participants reported a psychopathology level that was similar to that of community samples. Changes in psychopathology after bariatric surgery have been studied, with contradictory results: whereas some studies show improvements, others report increased levels of psychopathology. In the present study, no statistically significant changes were observed over time, suggesting that changes related to bariatric surgery do not have impacts on mental health. In this sense, psychopathology does not seem to be an important dimension to work with in these patients.

Contrary to our expectations, our sample showed significant differences over time in the openness, extraversion, and conscientiousness personality dimensions, which increased (medium effect size) over the time elapsed between surgery and the 12-month follow-up evaluation. Recent research has admitted that personality traits change in adulthood ${ }^{25,32}$ and that environmental factors and life experiences can play an important role in developing and changing personality. For example, Roberts \& Mroezek $^{33}$ have suggested that success in work is associated with increased measures of dominance. Additionally, Calugi et al. ${ }^{34}$ found that a stable relationship is associated with high levels of family conscientiousness traits. According to the same authors, insecurity and unstable relationships are associated with increased neuroticism. In accordance with these recent findings, our results suggest that the experience of bariatric surgery improved the health and life expectancy and caused changes to personality traits. However, there no differences in neuroticism were observed over time.

Our study indicates that bariatric surgery induces weight loss and reduces health problems and health complaints. Given these improvements, one could argue that bariatric surgery can clearly benefit obese people in terms of health and quality of life. Despite divergent data on the successful outcomes of bariatric surgery, our results suggest that the procedure can improve the health of the patients. Notwithstanding, taking into consideration individual and treatment differences, it is important to define success based on each patient's biological, physical, and psychological characteristics, as well as on the advantages potentially resulting from bariatric surgery (i.e., weight loss, reduction of comorbidities, and improved quality of life).

One of the limitations of our study is our small sample size. Also, we have not assessed eating disorders and pathological eating behaviors that are common in this population, especially after surgery. ${ }^{34}$ This assessment would require a control group to compare changes taking place over a period of time. 
Another important aspect of our data is that the patients here assessed had a peculiar characteristic: they were capable of mobilizing resources to ask for help and adopt support strategies. Further study should focus on obese persons who do not seek help and are incapable of pursuing their objectives.

Finally, despite evaluating patients at three different moments, the literature suggests that the less favorable outcomes of bariatric surgery begin to emerge 12 to 24 months after surgery. In this sense, it would be appropriate to continue evaluating the present sample, in order to confirm whether the improvements observed would be maintained over the years and to analyze any new difficulties that might arise.

We could not compare our results on personality dimensions with data from the community reference study, as these data are not available for the Portuguese version of the questionnaire. We expect that future studies will explore personality dimensions and the potential relations between personality dimensions and successful outcomes of the surgery. Furthermore, it would be useful to use more qualitative methods to understand the meanings attributed by individuals to bariatric surgery, patients' eating behaviors and the difficulties and needs of the treatment.

In spite of the limitations above, we do believe that the results of this study have implications for the future treatment of obesity. According to international guidelines, almost all morbidly obese patients should be considered candidates for bariatric surgery, but it is also important to evaluate their capacity to commit to the demands of the treatment. Eating seems to be an important strategy for dealing with problems and emotions, as well as an important source of social interaction and pleasure. After surgery, this behavior will have to be significantly restricted. This restriction, in turn, may promote new and different coping strategies.

In summary, these patients should be considered chronic. They should be continuously monitored according to their individual needs, and should be evaluated by an interdisciplinary team to promote lifestyle changes, maintain weight loss, and generally improve health. In cases of treatment failure, it is essential to understand what went wrong, consider other alternatives, always with a focus on the continued empowerment of patients.

\section{References}

1. World Health Organization. Special issue - Diet, nutrition and the prevention of chronic diseases: scientific background papers of the joint WHO/FAO Expert Consultation, Geneva, 28 January - 1 February 2002. Geneva: WHO; 2004. http://www.who.int/nutrition/ publications/obesity/PHNvol7no1afeb2004/en/index.html.

2. Finucane MM, Stevens GA, Cowan MJ, Danaei G, Lin JK, Paciorek $\mathrm{C}$, et al. National, regional, and global trends in body-mass index since 1980: systematic analysis of health examination surveys and epidemiological studies with 960 country-years and 9.1 million participants. Lancet. 2011;377:557-67.

3. Fried M, Hainer V, Basdevant A, Buchwald $H$, Deitel M, Finer $\mathrm{N}$, et al. Interdisciplinary European guidelines for surgery for severe (morbid) obesity. Obes Surg. 2007;17:260-70.

4. McEwen LN, Coelho RB, Baumann LM, Bilik D, Nota-Kirby B, Herman WH. The cost, quality of life impact, and cost-utility of bariatric surgery in a managed care population. Obes Surg. 2010;20:919-28

5. Chantler PD, Lakatta EG. Role of body size on cardiovascular function: can we see the meat through the fat? Hypertension. 2009;54:459-61.

6. Torgerson JS, Sjöström L. The Swedish obese subjects (SOS) study - rationale and results. Int J Obes Relat Metab Disord. 2001;25(Suppl 1):S2-4.

7. Sjöström L, Narbro K, Sjöström CD, Karason K, Larsson B, Wedel $H$, et al. Effects of bariatric surgery on mortality in Swedish obese subjects. N Engl J Med. 2007;357:741-52.

8. Sullivan M, Karlsson J, Sjöström L, Backman L, Bengtsson C, Bouchard C, et al. Swedish obese subjects (SOS) - an intervention study of obesity. Baseline evaluation of health and psychosocial functioning in the first 1743 subjects examined. Int J Obes Relat Metab Disord. 1993;17:503-12.

9. Abilés V, Rodríguez-Ruiz S, Abilés J, Mellado C, García A, Pérez de la Cruz A, et al. Psychological characteristics of morbidly obese candidates for bariatric surgery. Obes Surg. 2010;20:161-7.

10. Black DW, Goldstein RB, Mason EE. Prevalence of mental disorder in 88 morbidly obese bariatric clinic patients. Am J Psychiatry. 1992;149:227-34.

11. Kalarchian MA, Marcus MD, Levine MD, Soulakova JN, Courcoulas AP, Wisinski MS. Relationship of psychiatric disorders to 6-month outcomes after gastric bypass. Surg Obes Relat Dis. 2008;4:544-9.

12. Wadden TA, Stunkard AJ. Handbook of obesity treatment. London: The Guildford Press; 2004.

13. Mitchell JE, Zwaan M. Bariatric surgery: a guide for mental health professional. New York: Routledge; 2005.

14. Guisado JA, Vaz FJ. Personality profiles of the morbidly obese after vertical banded gastroplasty. Obes Surg. 2003;13:394-8.

15. Castelnuovo-Tedesco P. Psychological consequences of physical defects: a psychoanalytic perspective. Int Rev Psychoanal. 1981;8:145-54.

16. Castelnuovo-Tedesco P. Bypass surgery for superobesity. In: Blacker RS, editor. The psychological experience of surgery. New York: John Wiley; 1987.

17. Bruch H. Eating disorders: obesity, anorexia nervosa, and the person within. New York: Basic Books; 1979.

18. Hutzler JC, Keen J, Molinari V, Carey L. Super-obesity: a psychiatric profile of patients electing gastric stapling for the treatment of morbid obesity. J Clin Psychiatry. 1981;42:458-62.

19. Petry NM, Barry D, Pietrzak RH, Wagner JA.. Overweight and obesity are associated with psychiatric disorders: results from the National Epidemiologic Survey on Alcohol and Related Conditions. Psychosom Med. 2008;70:288-97.

20. Silva SS, Maia AC. Adverse childhood experiences, psychological characteristics and physical health problems: comparison among obese and non-obese. Rev Psiquiatr Clin. 2011;38:194-200.

21. Larsen F. Psychosocial function before and after gastric banding surgery for morbid obesity. A prospective psychiatric study. Acta Psychiatr Scand Suppl. 1990;359:1-57.

22. de Haes JC, van Knippenberg FC, Neijt JP. Measuring psychological and physical distress in cancer patients: structure and application of the Rotterdam Symptom Checklist. Br J Cancer. 1990;62:1034-8.

23. Canavarro C. Brief Symptom Inventory. Testes e provas psicológicas em Portugal. Braga: APPORT; 1999.

24. Derogatis LR, Unger R. Symptom Checklist-90-Revised. In: Weiner IB, Craighead WE, editors. The Corsini encyclopedia of psychology. Hoboken: John Wiley \& Sons; 2010.

25. Costa PT Jr, McCrae RR. Age changes in personality and their origins: comment on Roberts, Walton, and Viechtbauer (2006). Psychol Bull. 2006;132:26-8.

26. Green S, Salkind N. Using SPSS for Window and Macintosh: analyzing and understanding data. 5th ed. New Jersey: Pearson Prentice Hall; 2008.

27. Byrne S, Cooper Z, Fairburn C. Weight maintenance and relapse in obesity: a qualitative study. Int J Obes Relat Metab Disord. 2003;27:955-62.

28. Nelbom B, Naver L, Ladelund S, Hornnes N. Patient characteristics associated with a successful weight loss after bariatric surgery. Bariatr Nurs Surg Patient Care. 2010;5:313-9. 
29. Belanger SB, Wechsler FS, Nademin ME, Virden TB. Predicting outcome of gastric bypass surgery utilizing personality scale elevations, psychosocial factors, and diagnostic group membership. Obes Surg. 2010;20:1361-71.

30. Ray EC, Nickels MW, Sayeed S, Sax HC. Predicting success after gastric bypass: the role of psychosocial and behavioral factors. Surgery. 2003;134:555-63.

31. Kalarchian MA, Marcus MD, Levine MD, Courcoulas AP, Pilkonis $\mathrm{PA}$, Ringham RM, et al. Psychiatric disorders among bariatric surgery candidates: relationship to obesity and functional health status. Am J Psychiatry. 2007;164:328-34.

32. Roberts BW, Walton K, Bogg T, Caspi A. De-investment in work and non-normative personality trait change in young adulthood. Eur J Pers. 2006;20:461-74.
33. Roberts B, Mroezek D. Personality trait change in adulthood. Curr Dir Psychol Sci. 2008;17:31-5.

34. Calugi S, Dalle Grave R, Marchesini G. Night eating syndrome in class II-III obesity: metabolic and psychopathological features. Int J Obes (Lond). 2009;33:899-904.

\section{Correspondence:}

Susana Sofia Pereira da Silva

Scholl of Management and Industrial Studies

Rua D. Sancho I, 981

4480-876 - Vila do Conde - Portugal

Tel./Fax: +35-12-5229-1700/1714

E-mail: susanasofiapsilva@gmail.com 